\title{
TEMPERATURE VARIATION WITH LATITUDE IN THE UPPER SOLAR PHOTOSPHERE: RELEVANCE TO SOLAR OBLATENESS MEASUREMENTS AND FACULAR MODELS
}

\author{
ANDREW P. INGERSOLL \\ Division of Geological and Planetary Sciences, California Institute of Technology, Calif., U.S.A. \\ and \\ GARY A. CHAPMAN \\ San Fernando Observatory, Space Physics Laboratory, The Aerospace Corporation
}

(Received 8 October, 1974; revised 25 February, 1975)

\begin{abstract}
Altrock and Canfield's observations of temperature variation with latitude in the upper solar photosphere refer to higher levels (smaller optical depths) than those to which Dicke and Goldenberg's solar oblateness observations refer. These higher levels account for only $1 \%$ of Dicke and Goldenberg's observed intensity. Thus Altrock and Canfield's observations are not inconsistent with models which have been proposed to account for solar oblateness observations by means of a brightness variation with solar latitude.
\end{abstract}

\section{Introduction}

Altrock and Canfield (1972a) observed the intensity of emitted radiation as a function of solar latitude during the period 15-22 June 1971, using a spectral region of $30 \mathrm{~m} \AA$ width centered $0.77 \AA$ to the red of $\mathrm{Ca} I \mathrm{II} \mathrm{K}_{3}$. The radiation was shown to originate at $\tau \simeq 10^{-3}$, where $\tau=\tau_{5}$, the optical depth at $5000 \AA$ wavelength. The observations imply a drop in the mean temperature at $\tau \simeq 10^{-3}$ of almost $10 \mathrm{~K}$ from active-region latitudes to polar regions.

Using these data from 1971, Canfield (1973) attempted to estimate the equator-topole temperature difference during 1966, the period when Dicke and Goldenberg's (1967) solar oblateness observations were made. He assumed that temperature differences are linearly related to photospheric magnetic field intensities, and used magnetic field data from 1966 to infer an upper limit of several degrees to the equatorial temperature excess at $\tau \simeq 10^{-3}$ in 1966 .

However, the relevance of these inferences to solar oblateness studies is questionable. First, the assumption that the relation between temperature differences and magnetic field is linear over different parts of the solar cycle is somewhat speculative. Comparing Figures 2, 3 and 4 of Stenflo (1972), the magnetic field is seen to fluctuate with higher frequency and greater relative amplitude change than faculae or sunspots, for example.

Second and more important, the region of overlap between Altrock and Canfield's observations and Dicke and Goldenberg's is small. The solar oblateness observations were made through a broad-band filter centered at $7000 \AA$. Light from the outermost portion of the solar disk, for which $0<\mu \lesssim 0.1$, contributed to the detection. Thus the solar oblateness observations refer to the region $\tau_{7} \lesssim 0.1$, where $\tau_{7} \simeq 1.25 \tau$ is the optical 
depth at $7000 \AA$ (Gingerich and de Jager, 1968; Dicke, 1973). In contrast, Altrock and Canfield's observations refer only to $\tau \lesssim 10^{-3}$.

Several models (Ingersoll and Spiegel, 1971; Durney and Werner, 1971; Chapman and Ingersoll, 1972, 1973) have been proposed to explain the solar oblateness observations in terms of an equator-to-pole temperature difference (see Dicke 1972, 1973, 1974 for replies). These models all contain the feature that a relatively large equatorto-pole temperature difference is required if it is confined to relatively small values of $\tau$, e.g., $\tau<10^{-3}$, but that only a small temperature difference is required if it is distributed uniformly with respect to $\tau$ in the range $0<\tau<0.1$.

Thus Canfield's analysis, which suggests a small value for the equator-to-pole temperature difference at $\tau \simeq 10^{-3}$, is not necessarily inconsistent with models of brightness variation as a cause of solar oblateness. The region $0<\tau<10^{-3}$ contributes only about $1 \%$ of the intensity observed at $\mu \simeq 0.1$, so a small equator-to-pole temperature difference at $\tau \simeq 10^{-3}$ can be easily offset by a slightly larger temperature difference at $10^{-3}<\tau<0.1$.

Most of the models that have been proposed to explain the solar oblateness observations as a brightness variation can be adjusted slightly to agree with Altrock and Canfield's observations at $\tau \simeq 10^{-3}$. Moreover, the models can be made to agree even if the temperature difference at all levels never exceeds that observed at $\tau \simeq 10^{-3}$. All that is required is that these temperature differences persist to sufficiently great optical depths, e.g., to $\tau=0.1$. In the next section we consider the horizontally homogeneous models, in which the assumed brightness variation is a smooth function of solar latitude. In later sections we consider models in which the equatorial excess brightness is concentrated in isolated facular patches approximately $750 \mathrm{~km}$ in diameter.

\section{Horizontally Homogeneous Models}

There is very little difference between the models of Durney and Werner (1971) and Ingersoll and. Spiegel (1971). Both assume an equator-to-pole temperature difference $\Delta T$ that depends on $\tau$ and has its greatest amplitude in the region $0<\tau<0.1$. The value of the integral of $\Delta T(\tau)$ with respect to $\tau$ defines those models which could explain Dicke and Goldenberg's data. The basic formalism is given by Ingersoll and Spiegel. Durney and Werner give explicit calculations for a variety of distributions $\Delta T(\tau)$, with emphasis on those that do not vanish at large values of $\tau$. Since we are concerned here with the region $\tau \simeq 10^{-3}$ these differences will be ignored in what follows.

As shown by Ingersoll and Spiegel, a temperature difference $\Delta T(\tau)$ satisfying

$$
\int_{0}^{\infty} E_{2}\left(\tau_{7} / \mu_{0}\right) \Delta B_{7}(\tau) \mathrm{d} \tau_{7}=\left(1 / \mu_{0}\right) I_{7}\left(\mu_{0}\right)(\Delta r / r),
$$

is capable of explaining Dicke and Goldenberg's data. Here $B_{7}$ and $\tau_{7}$ are the Planck function and optical depth, respectively, at a wavelength of $7000 \AA$, and $\Delta B_{7}(\tau)$ is the equator-to-pole difference of $B_{7}$ associated with $\Delta T(\tau) . E_{2}(x)$ is the second expo- 
nential integral. $\mu_{0}$ is the cosine of the emission angle at the innermost edge of Dicke and Goldenberg's measurement zone, and $I_{7}\left(\mu_{0}\right)$ is the corresponding photospheric intensity at $7000 \AA . \Delta r / r$ is the value of the excess solar oblateness, corrected for solar surface rotation, that was inferred by Dicke and Goldenberg from their measurements.

We are not considering Dicke and Goldenberg's $5300 \AA$ observations. These observations in the green involve only 6 days and were obtained at the end of the 1966 observing season (Dicke and Goldenberg, 1974). Dicke (1973) and Dicke and Goldenberg (1974) have maintained that there is no color difference in their oblateness measurements. However, since the green observations occur at the end of the 1966 season they can not be examined for the seasonal trend in $\sin 2 P$. Without the seasonal trend, one can not determine a reliable value for the oblateness in the green. Further, there are no published data where green and red observations were obtained on the same day. Thus we do not feel that a lack of color difference in the Princeton data is definitely established.

For the three values $\mu_{0}=0.12,0.16$ and 0.20 for which data were taken, the right side of Equation (1) is approximately independent of $\mu_{0}$ and equal to $1.3 \times 10^{-4} I_{7}(1)$, where $I_{7}(1)$ is the intensity at the center of the solar disk at $7000 \AA$. Equation (1) can then be rewritten

$$
\int_{0}^{\infty} E_{2}\left(\frac{\tau_{7}}{\mu_{0}}\right) \frac{\mathrm{d} B_{7}(T)}{\mathrm{d} T} \Delta T \frac{\mathrm{d} \tau_{7}}{\mathrm{~d} \tau} \mathrm{d} \tau=1.3 \times 10^{-4} I_{7}(1) .
$$

Here $\Delta T(\tau)$ is assumed to be small enough so that the Planck function change can be approximated by its derivative. Moreover the variable of integration is now $\tau$ instead of $\tau_{7}$. The functions $T(\tau)$ and $\tau_{7}(\tau)$ refer to the mean photosphere, and are obtained from a standard model (Gingerich and de Jager, 1968).

As shown by Ingersoll and Spiegel, Equation (2) can be satisfied for all $\mu_{0}$ if $\Delta T(\tau)$ is non-zero only where $\tau_{7} / \mu_{0} \ll 1$, so that $E_{2}\left(\tau_{7} / \mu_{0}\right) \simeq 1$. The contribution to the integral then comes entirely from the region near the temperature minimum, where $T \simeq 4600 \mathrm{~K}$ and $\mathrm{d} \tau_{7} / \mathrm{d} \tau \simeq 1.25$. This yields

$$
\int_{0}^{\tau_{0}} \Delta T(\tau) \mathrm{d} \tau \simeq 0.32 \mathrm{~K}
$$

where $\tau_{0}$ is the value of $\tau$ beyond which $\Delta T$ is assumed to vanish $\left(\Delta T=0\right.$ for $\left.\tau>\tau_{0}\right)$, with $\tau_{0} \lesssim 0.01$ in order that $E_{2}\left(\tau_{7} / \mu_{0}\right) \simeq 1$.

Equation (3) gives the middle curve in Figure 2 of Ingersoll and Spiegel, and provides a good fit to Dicke and Goldenberg's data. The numberical constant $0.32 \mathrm{~K}$ is slightly larger than the value $0.30 \mathrm{~K}$ given by Ingersoll and Spiegel. The change 1eflects mainly the use of intensity at $7000 \AA$ instead of integrated intensity. Dicke (1973) and Durney and Werner (1971) consider cases where $E_{2}\left(\tau_{7} / \mu_{0}\right)<1$, and so they require slightly larger values of $\Delta T$ to fit the data. In addition, Durney and Werner (1971) do not correct for solar surface rotation. Nevertheless, all of the above analyses are in approximate agreement. 
Most distributions satisfying Equation (3) with $\tau_{0} \simeq 10^{-2}$ may be modified slightly so as to agree with Altrock and Canfield's observations at $\tau \simeq 10^{-3}$. For example, the two distributions

and

$$
\Delta T=32 \mathrm{~K} \quad\left(0 \leqslant \tau \leqslant 10^{-2}\right),
$$

$$
\Delta T=32 \mathrm{~K} \quad\left(0.3 \times 10^{-2} \leqslant \tau \leqslant 1.3 \times 10^{-2}\right),
$$

with $\Delta T=0$ for all other values of $\tau$, both satisfy Equation (3) although they differ greatly at $\tau<10^{-3}$. The second distribution is a slightly modified form of the first, chosen to be consistent with Altrock and Canfield's low value of $\Delta T$ within a decade in $\tau$ about the point $\tau \simeq 10^{-3}$.

The case opposite to (4), where $\Delta T$ is constant down to large values of $\tau$, is marginally inconsistent both with Altrock and Canfield's (1972b) continuum observations and with Dicke and Goldenberg's (1967) oblateness data. For instance, with $\Delta T=4.0 \mathrm{~K}$ for all $\tau$, the left side of (2) is equal to the right side at $\mu_{0}=0.16$, but is equal to (1.0 and 1.6) $\times 10^{-4} I_{7}(1)$ for $\mu_{0}=0.12$ and 0.20 , respectively. This dependence on $\mu_{0}$ is illustrated by the lower curve in Figure 2 of Ingersoll and Spiegel, and is a poor fit to Dicke and Goldenberg's data. Moreover, this value of $\Delta T$ is marginally inconsistent with Altrock and Canfield's (1972b) continuum results for $\mu \geqslant 0.2(\tau \gtrsim 0.2)$ which give $\Delta T \approx-1.5 \pm 4 \mathrm{~K}$. The formal error of these observations is $0.6 \mathrm{~K}$, but the authors do not rule out possible systematic errors of 3-4 K. Active regions were excluded from their analysis, so the results may not be directly applicable to the present discussion.

The objections which have been raised against these horizontally homogeneous models concern limb brightening at the equator and the supply of energy to the region $\tau \lesssim 0.01$. The first objection is less serious than the second. For example, $T(\tau)$ in the standard photospheric model passes through the point $T=4630 \mathrm{~K}, \tau \simeq 0.0125$. We can satisfy Equation (3) by using the standard model at the poles and a modified standard model with $T=4630 \mathrm{~K}=$ constant for $\tau \leqslant 0.0125$ at the equator. This gives a temperature excess at the equator of $30 \mathrm{~K}$ as $\tau \rightarrow 0$, but no region of temperature inversion and no limb brightening, as observed.

The question of energy supply to the region $\tau \lesssim 0.01$ has been discussed elsewhere (Durney and Roxburgh, 1969; Ingersoll and Spiegel, 1971; Durney and Werner, 1971; Dicke, 1973). Large-scale processes, e.g., meridional circulations, variations in the mechanical heating of the lower chromosphere, etc., do not seem capable of maintaining temperature differences of the magnitude implied by Equation (3). However, smallscale regions of anomalous intensity, e.g., faculae and sunspots, are known to exist. And although their energy supply is not well understood, their contribution to Dicke and Goldenberg's signal can be estimated from observation, as discussed in the next section.

\section{A Facular Brightness Model}

Chapman and Ingersoll (1972) showed that a simple model in which faculae are assumed to be optically thin patches above the top of the photosphere is consistent with 
observations of facular limb-darkening and reproduces the $\mu_{0}$-dependence of Dicke and Goldenberg's signal. They also showed that the equatorial flux excess due to faculae is sufficient to account quantitatively for Dicke and Goldenberg's signal. The latter calculation was based on estimates of facular area and latitude distribution from Allen (1963). A similar calculation indicates that the contribution of sunspots is small. Here we show that Chapman and Ingersoll's simple facular model is also consistent with Altrock and Canfield's data.

We assume that the facular source region lies entirely above the solar limb $(\tau<$ $<0.004)$. For simplicity, temperature is assumed to be constant in this source region, and is typically about $6500 \mathrm{~K}$. The diameter of the source region is $750 \mathrm{~km}$, and the source region is optically thin for both vertical and horizontal rays. The optical depth scale height is $100 \mathrm{~km}$ within the facula.

The model is made to be consistent with observations of facular contrast vs. viewing angle at $5500 \AA$ wavelength (Rogerson, 1961; Chapman, 1970). For a ray with emission angle $\cos ^{-1} \mu$, the maximum optical thickness through the facula at $5500 \AA$ wavelength is

$$
\tau_{55}^{\prime}=\frac{\tau_{55}^{*}}{\mu}\left[1-\exp \left(-\frac{D}{H} \frac{\mu}{\sqrt{1-\mu^{2}}}\right)\right]
$$

Here $\tau_{55}^{*}$ is the value of the $5500 \AA$ optical depth at the base of the facular source region, $D$ is the diameter and $H$ is the optical depth scale height of the facula. The maximum emergent intensity $I_{55}(\mu)$ of the facula is

$$
I_{55}(\mu)=\bar{I}_{55}(\mu) \exp \left(-\tau_{55}^{\prime}\right)+B_{55}\left(T_{\mathrm{f}}\right)\left[1-\exp \left(-\tau_{55}^{\prime}\right)\right]
$$

where $\bar{I}_{55}(\mu)$ is the intensity of the underlying photosphere (Gingerich and de Jager, $1968)$ and $B_{55}\left(T_{\mathrm{f}}\right)$ is the Planck function evaluated at the temperature $T_{\mathrm{f}}$ of the facular source region. We define

$$
\frac{I_{55}(\mu)}{I_{55}(1)}, \text { and } \frac{I_{55}(\mu)-\bar{I}_{55}(\mu)}{I_{55}(\mu)},
$$

as the normalized facular brightness and facular contrast, respectively, at $5500 \AA$

TABLE I

Facular models and associated intensity variation at $3934 \AA$

\begin{tabular}{lllll}
\hline $\begin{array}{l}T \\
(\mathrm{~K})\end{array}$ & $\left(\tau^{*}\right)_{5000 \AA}$ & $(\tau \Delta B / I)_{\text {ro00 }} \AA$ & $\begin{array}{l}\bar{A}(1966) \\
\left(\times 10^{-3}\right)\end{array}$ & $(\Delta I / I)_{3934 \AA}$ \\
\hline & & & & \\
6200 & 0.0665 & 0.0494 & 1.37 & 0.0170 \\
6400 & 0.0519 & 0.0463 & 1.46 & 0.0224 \\
6600 & 0.0420 & 0.0442 & 1.53 & 0.0285 \\
6800 & 0.0349 & 0.0425 & 1.59 & 0.0354 \\
7000 & 0.0296 & 0.0411 & 1.64 & 0.0430 \\
\hline
\end{tabular}


wavelength. Finally, we let $\tau^{*}=\tau_{55}^{*} / 1.08$ be the optical depth of the facular source region at the standard wavelength of $5000 \AA$.

With $D / H=7.5$, the only undetermined parameters are $\tau^{*}$ and $T_{\mathrm{f}}$, but the facular contrast observations require that only one of these is independent. Columns 1 and 2 of Table I give pairs of values $T_{\mathrm{f}}$ and $\tau^{*}$ which fit the observations. Figure 1 shows

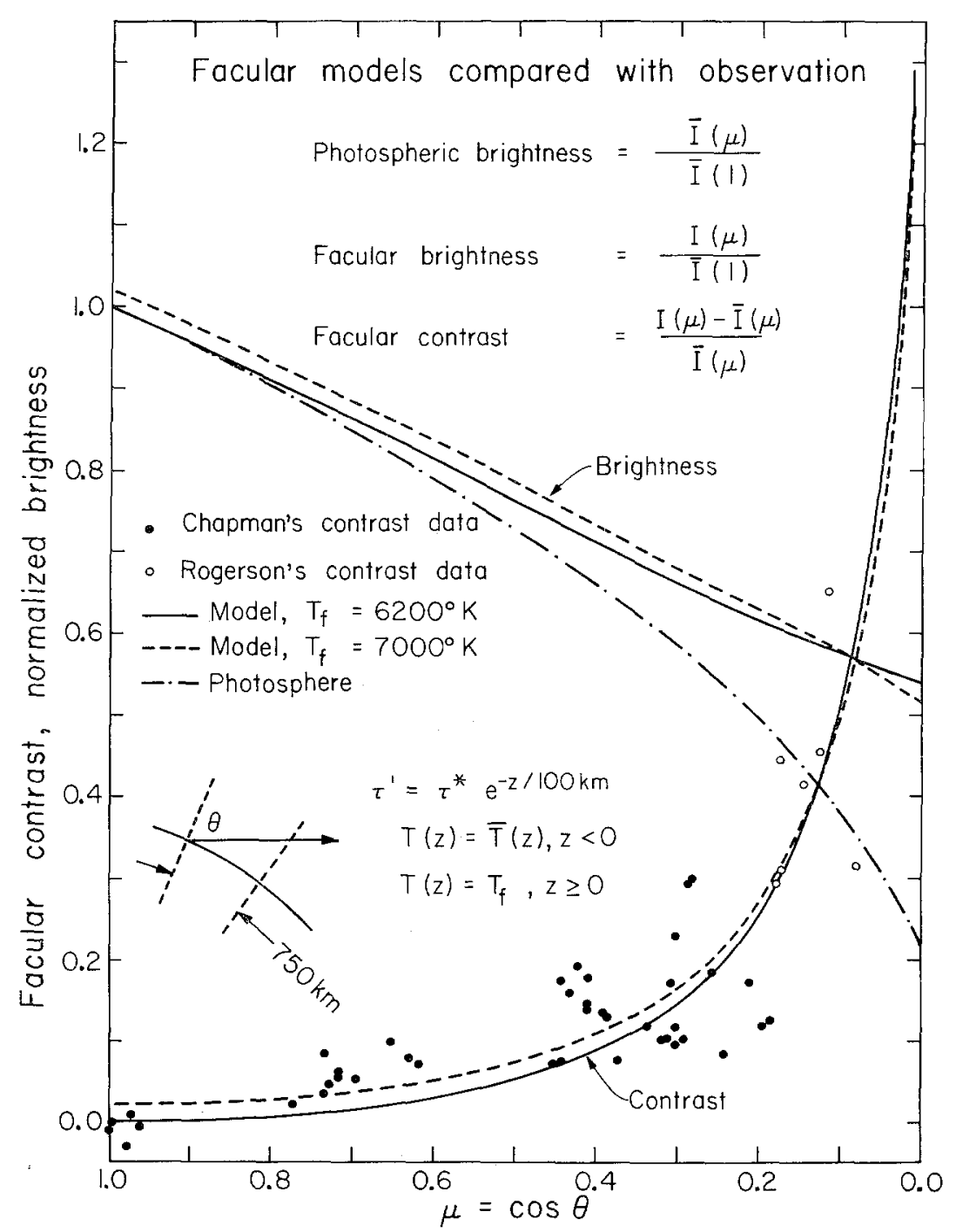

Fig. 1. Facular contrast, normalized facular brightness and photospheric brightness vs. viewing angle at $5500 \AA$ wavelength. Observations of facular contrast are from Chapman (1970) and Rogerson (1961). Photospheric brightness is from Gingerich and de Jager (1968). Model calculations of facular brightness and facular contrast, described in the text and in Table $\mathrm{I}$, are for $T_{\mathrm{f}}=6200 \mathrm{~K}$ and $7000 \mathrm{~K}$, where $T_{\mathrm{f}}$ is the temperature of the facular source region. Note that facular brightness decreases as $\mu \rightarrow 0$ whereas facular contrast increases. This facular model can account for Dicke and Goldenberg's (1967) excess solar oblateness and is also consistent with Altrock and Canfield's (1972) observations. 
normalized facular brightness and facular contrast at $5500 \AA$ for $T_{\mathrm{f}}=6200 \mathrm{~K}$ and $T_{\mathrm{f}}=7000 \mathrm{~K}$. Curves for the other values of $T_{\mathrm{f}}$ listed in Table 1 lie between the curves shown in the figure. The observations cover the range $1 \geqslant \mu \geqslant 0.07$, and the agreement is satisfactory.

Note that for all five models the optical depth $\tau^{*}$ is sufficiently small that the facula is optically thin at $7000 \AA$ for all emergent rays. This can be demonstrated by computing $\tau_{7}^{\prime}$ using the method of Equation (5). The fact that faculae are optically thin ensures that the facular contribution to Dicke and Goldenberg's signal is proportional to $\mu_{0}$ (middle curve in Figure 2 of Ingersoll and Spiegel, 1971; curve 1 in Figure 1 of Dicke, 1973). This $\mu_{0}$ dependence is consistent with Dicke and Goldenberg's data before correction for faculae (Dicke, 1973), and it is difficult to see how a correction for faculae could change this dependence. Thus Dicke's (1973) correction for faculae implies a different (stronger) dependence of facular signal on $\mu_{0}$ than the model presented here.

According to Figure 1, facular brightness decreases almost linearly with $\mu$ from $\mu=1$ to $\mu=0$. This is due to the fact that the limb darkening of the photospheric background more than offsets the increased contribution from the optically thin facular region as the limb is approached. Thus, although facular limb darkening is much less than that of the surrounding photosphere, there is no limb brightening, according to this simple model. This fact largely removes one objection to the model discussed at the end of Section II in Chapman and Ingersoll (1972).

Overlap between two or more faculae in the field of view was not considered in the above model. This is consistent with the observations shown in Figure 1, which refer to isolated facular patches. Our model predicts substantial brightening whenever overlap occurs, but such effects have not been observed, perhaps because the number of high-resolution limb observations is so limited. However, as long as the total optical path along the line of sight through facular material is small, overlap would not have affected Dicke and Goldenberg's measurement of total excess flux.

Column 3 of Table I gives values of

$$
\tau_{7}^{*}\left[B_{7}\left(T_{\mathrm{f}}\right)-\bar{I}_{7}\left(\mu_{0}\right)\right] / \bar{I}_{7}(1), \quad \mu_{0}=0.1,
$$

where the subscript 7 refers to $7000 \AA$ wavelength, with $\tau_{7}^{*}=1.25 \tau^{*}$. These values are to be compared with the quantity $\tau_{0} \Delta B / I_{0} \simeq 0.03$ obtained by Chapman and Ingersoll using wavelength-integrated intensities. As shown in that paper, this quantity is the contribution per unit area of faculae to Dicke and Goldenberg's observed signal.

Chapman and Ingersoll (1972) show how the facular brightness estimate $\tau_{0} \Delta B / I_{0}$ may be combined with $\bar{A}(66)$, the fraction of the solar surface covered by faculae in 1966 , to yield an equivalent oblateness due to faculae. Their result reduces to

$$
1.922 \frac{\tau_{0} \Delta B}{I_{0}} \bar{A}(66)=\frac{1}{\mu_{0}} \frac{\bar{I}\left(\mu_{0}\right)}{I(1)} \frac{\Delta r}{r} \simeq 1.3 \times 10^{-4},
$$

where the last step represents a fit to Dicke and Goldenberg's data as in Equations (1) 
and (2) of the present paper. We shall use (9) to compute the value of $\bar{A}(66)$ necessary to account for all of Dicke and Goldenberg's signal, and shall show that this value of facular area is not inconsistent with Altrock and Canfield's (1972a) observations. Statistical arguments based on Chapman and Ingersoll's (1972) daily facular signals are largely irrelevant here, for two reasons. First, the absolute amplitude of the daily facular signals is only poorly known, and second, the daily facular signals are insensitive to a possible background of weak faculae that might easily have contributed both to Dicke and Goldenberg's and Altrock and Canfield's observations. We feel that the amount of correlation between our daily facular signal and Dicke and Goldenbetg's daily signal is consistent with the hypothesis that faculae contributed all of their excess solar oblateness. However, Dicke disagrees, so we refer the reader to recent papers on both sides of this argument (Chapman and Ingersoll, 1973; Dicke, 1973, 1974; Dicke and Goldenberg, 1974).

Values of $\bar{A}(66)$ computed from (9) are given in column 4 of Table I, where the corresponding values of $\tau_{0} \Delta B / I_{0}$ from column 3 have been used. These values of $\bar{A}(66)$ are somewhat smaller than the value $1.7 \times 10^{-3}$ which Chapman and Ingersoll obtained from $\$ 87$ of Allen (1963). The difference is not significant, however, because of the different ways the two estimates were obtained. In fact, Allen's estimate, combined with $\tau_{0} \Delta B / I_{0}$ from the facular model of this paper, gives an oblateness signal due to faculae which is larger than that measured by Dicke and Goldenberg, according to (9).

These results are consistent with facular areas and contrasts which we obtained from photographs of the Sun in 1966. As pointed out at the bottom of p. 1019 of Chapman and Ingersoll (1973), the facular areas obtained from photographs were higher, and the facular contrasts lower by a factor of 3 compared with the numbers in Table I, but the net flux excess due to faculae was about the same as in (9). The difference in facular areas can be accounted for by the lower resolution of the images used in our photographic study. Finally, the facular area estimates obtained from (9) are consistent with Altrock and Canfield's contrast measurements at $3934 \AA$, as we now demonstrate.

\section{Comparison with Altrock and Canfield's Observations}

Altrock and Canfield observed an intensity difference between active region latitudes and the poles in 1971 of about $1.9 \%$, which they express as a mean temperature difference of about $10 \mathrm{~K}$. Here we show that the facular models of Table I give values of the mean intensity difference ranging from 1 to $4 \%$ at the wavelength used in Altrock and Canfield's observations.

As an estimate of mean facular area for June 1971, we shall scale the numbers $\bar{A}(66)$ in column 4 of Table I by $R_{\mathrm{Z}}$, the Zürich sunspot number. Thus we take

$$
\bar{A}(71)=\bar{A}(66)(67 / 55),
$$

where the numbers 67 and 55 are the values of $R_{\mathrm{Z}}$ for June 1971 and August 1966, 
respectively (Solar Geophysical Data, 1974). We shall assume that in 1971 faculae were distributed as $\sin ^{2} \theta$, where $\theta$ is solar colatitude. This distribution roughly follows Altrock and Canfield's excess brightness distribution. With proper normalization this becomes

$$
A(\theta)=\frac{3}{2} \bar{A} \sin ^{2} \theta
$$

where $\bar{A}$ is $\bar{A}(71)$ obtained from Equation (10). This distribution reaches its maximum value at the equator, $\theta=90^{\circ}$, whereas Altrock and Canfield's measured excess brightness reaches its maximum value at active region latitudes, $\theta=75^{\circ}$. To be consistent, we shall use the maximum intensity difference (i.e., between $\theta=0$ and $\theta=90^{\circ}$ ) implied by the distribution (11) in comparing with Altrock and Canfield's data. Since $\sin 75^{\circ}=$ $=0.966$, the difference is small in any case.

The mean temperature of the photosphere at $\tau \simeq 10^{-3}$ is about $4600 \mathrm{~K}$. Therefore the maximum latitudinal intensity variation associated with faculae at temperature $T_{\mathrm{f}}$ and area $A(\theta)$ is

$$
\frac{\Delta I}{I}=\left[\frac{B_{\lambda}\left(T_{\mathrm{f}}\right)}{B_{\lambda}(4600)}-1\right]\left[A\left(\frac{\pi}{2}\right)-A(0)\right] .
$$

The Planck function $B_{\lambda}$ is evaluated at $\lambda=3934 \AA$, corresponding to the Ca II K line. Values of $\Delta I / I$ are given in column 5 of Table $\mathrm{I}$. The model with $T_{\mathrm{f}}=6300 \mathrm{~K}$ gives the value of $\Delta I / I$ that is most in accord with Altrock and Canfield's observed value.

In other words, the simple facular model of Table I, which gives the observed value of Dicke and Goldenberg's oblateness signal for 1966, is also consistent with Altrock and Canfield's observed value of the equatorial brightening on the wings of the Ca II K line in 1971. Note, however, that the optical thickness $\tau^{*}$ of the facular source region is about 0.06 for $T_{\mathrm{f}}=6300 \mathrm{~K}$, whereas Altrock and Canfield only sampled the region $\tau \lesssim 10^{-3}$. Thus it is largely fortuitous that an isothermal facular model based on the mean brightness of the region $0<\tau<0.06$ should give consistent results when applied to the level $\tau \simeq 10^{-3}$. As with the homogeneous models, the region of overlap between Altrock and Canfield's observations and those of Dicke and Goldenberg is too small to permit detailed comparison.

\section{Acknowledgment}

This research was supported by company funds of The Aerospace Corporation and by the National Aeronautics and Space Administration under Grant NGL 05-002-003 awarded to the California Institute of Technology.

\section{References}

Allen, C. W.: 1963, Astrophysical Quantities, 2nd ed., Athlone Press, London.

Altrock, R. C. and Canfield, R. C.: 1972a, Astrophys. J. 171, L71.

Altrock, R. C. and Canfield, R. C.: 1972b, Solar Phys. 23, 257.

Canfield, R. C.: 1973, Astrophys. J. 179, 643.

Chapman, G. A.: 1970, Solar Phys. 14, 315. 
Chapman, G. A. and Ingersoll, A. P.: 1972, Astrophys. J. 175, 819.

Chapman, G. A. and Ingersoll, A. P.: 1973, Astrophys. J. 183, 1005.

Dicke, R. H.: 1972, Astrophys. J. 175, 831.

Dicke, R. H.: 1973, Astrophys. J. 180, 293.

Dicke, R. H.: 1974, Science 184, 419.

Dicke, R. H. and Goldenberg, H. M.: 1967, Phys. Rev. Lett. 18, 313.

Dicke, R. H. and Goldenberg, H. M.: 1974, Astrophys. J. Suppl. Ser. 27, 131.

Durney, B. R. and Roxburgh, I. W.: 1969, Nature 221, 646.

Durney, B. R. and Werner, N. E.: 1971, Solar Phys. 21, 21.

Gingerich, O. and de Jager, C.: 1968, Solar Phys. 3, 5.

Ingersoll, A. P. and Spiegel, E. A.: 1971, Astrophys. J. 163, 375.

Rogerson, J. B.: 1961, Astrophys. J. 134, 331.

Solar Geophysical Data: 1974, U.S. Department of Commerce, National Oceanic and Atmospheric Administration, compiled by the Aeronomy and Space Data Center, Boulder, Colorado.

Stenflo, J, O.: 1972, Solar Phys. 23, 307. 